\title{
Upgrades of Edge, Divertor and Scrape-Off Layer Diagnostics of W7-X for OP1.2
}

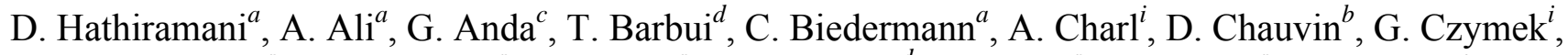
C.P. Dhard ${ }^{a}$, P. Drewelow ${ }^{a}$, A. Dudek ${ }^{a}$, F. Effenberg ${ }^{d}$, G. Ehrke $^{a}$, M. Endler ${ }^{a}$, D.A. Ennis ${ }^{g}$,

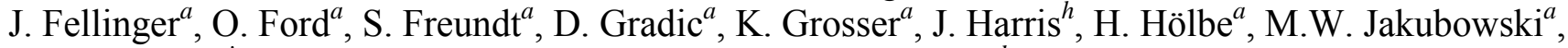
M. Knaup ${ }^{i}$, G. Kocsis ${ }^{c}$, R. König ${ }^{a}$, M. Krause ${ }^{a}$, T. Kremeyer ${ }^{d}$ P. Kornejew ${ }^{a}$, M. Krychowiak ${ }^{a}$, H.T. Lambertz ${ }^{i}$, H. Jenzsch ${ }^{a}$, M. Mayer ${ }^{\mathrm{e}}, \mathrm{S}$. Mohr $^{a}$, O. Neubauer ${ }^{i}$, M. Otte ${ }^{a}$, V. Perseo ${ }^{a}$, D. Pilopp ${ }^{a}$, L. Rudischhauser ${ }^{a}$, O. Schmitz ${ }^{d}$, B. Schweer ${ }^{i}$, M. Schülke ${ }^{a}$, L. Stephey ${ }^{d}$, T. Szepesi ${ }^{c}$, A. Terra ${ }^{i}$, M. Toth ${ }^{c}$, U. Wenzel ${ }^{a}$, G.A. Wurden ${ }^{f}$, S. Zoletnik ${ }^{c}$, T. Sunn Pedersen ${ }^{a}$ and the W7-X Team

\author{
${ }^{a}$ Max-Planck-Institut für Plasmaphysik, Greifswald, Germany \\ ${ }^{b}$ Commissariat à l'Energie Atomique DRF/IRFM, Cadarache, France \\ ${ }^{c}$ Wigner RCP RMI, Budapest, Hungary \\ ${ }^{d}$ Department of Engineering Physics, University of Wisconsin, Madison, USA \\ ${ }^{e}$ Max-Planck-Institut für Plasmaphysik, Garching, Germany \\ ${ }^{f}$ Los Alamos National Laboratory, Los Alamos, New Mexico, USA \\ ${ }^{g}$ University of Auburn, Auburn, AL, USA \\ ${ }^{\text {h}}$ Oak Ridge National Laboratory, TN, USA
}

${ }^{i}$ Forschungszentrum Jülich $\mathrm{GmbH}$, Institut für Energie- und Klimaforschung - Plasmaphysik, Jülich, Germany

\begin{abstract}
Wendelstein 7-X (W7-X) is the world's largest superconducting nuclear fusion experiment of the optimized stellarator type. In the first Operation Phase (OP1.1) helium and hydrogen plasmas were studied in limiter configuration. The heating energy was limited to $4 \mathrm{MJ}$ and the main purpose of that campaign was the integral commissioning of the machine and diagnostics, which was achieved very successfully. Already from the beginning a comprehensive set of diagnostics was available to study the plasma. On the path towards high-power, high-performance plasmas, W7-X will be stepwise upgraded from an inertially cooled (OP1.2, limited to $80 \mathrm{MJ})$ to an actively cooled island divertor (OP2, $10 \mathrm{MW}$ steady-state plasma operation). The machine is prepared for OP1.2 with 10 inertially cooled divertor units, and the experimental campaign has started recently.

The paper describes a subset of diagnostics which will be available for OP1.2 to study the plasma edge, divertor and scrape-off layer physics including those already available for OP1.1, plus modifications, upgrades and new systems. The focus of this summary will be on technical and engineering aspects, like feasibility and assembly but also on reliability, thermal loads and shielding against magnetic fields.
\end{abstract}

Keywords: Wendelstein 7-X, Diagnostics, Plasma edge, SOL, Steady-state operation.

\section{Introduction}

Wendelstein 7-X (W7-X) is the world's largest superconducting stellarator located in Greifswald, Germany [1]. On the path towards a stellarator fusion power plant [2], its goal is to demonstrate the feasibility and functionality of the optimized stellarator concept in steady state operation [3,4]. In December 2015 W7-X started its first operation phase (OP1.1) in limiter configuration. The main purpose of OP1.1 was the commissioning of the machine [5], but a successful physics program could already be conducted in parallel [6-8]. The final goal is a high-density high-power steady state operation with 30-minutes plasma pulses at an ECRH power of about $10 \mathrm{MW}$ while using an actively cooled divertor (OP2). The next step towards this milestone in operation will be 10 -second plasma pulses in hydrogen at about $8 \mathrm{MW}$ (OP1.2), using inertially cooled Test Divertor Units (TDU) [9,10]. Instead of $10 \mathrm{~s}$, longer pulses with less power resulting in the same heating energy of $80 \mathrm{MJ}$ are also possible during this operation phase. OP1.2 will be split into two phases OP1.2a and OP1.2b of about 3 months each. Scraper elements (section 4.10) will be installed between these two phases [11], see also section 4.10. A considerable set of diagnostics was already in operation for OP1.1 to study the edge physics [12]. For OP1.2 this set of diagnostics has been further improved and extended.

We do not focus on the aim of the individual diagnostics from the physics point of view, which is described elsewhere [12], but rather on engineering aspects, and on the challenges of the individual systems.

\section{General design remarks}

In a challenging and large project like W7-X, design and engineering aspects, requirements and boundary conditions for individual diagnostics are set by different sides like the authorities, the overall $\mathrm{W} 7-\mathrm{X}$ project and the diagnostic project itself. Technical guidelines valid for the overall W7-X project assist in the development and design process. For instance, a technical guideline 
for materials helps to select the right material with respect to mechanical properties, radiation demands, vacuum compatibility, Electron Cyclotron Resonance Heating $(\mathrm{ECRH})$ stray radiation, durability in contact with cooling water, fire protection, electrical properties, and allowed magnetic susceptibility. Usually such requirements also depend on the location where a component will be installed. For example, the introduced perturbation on the magnetic field of W7-X is depending on the magnetic susceptibility and the distance to the magnet system, or the choice of available materials is quite limited if they are possibly exposed to convective loads of the plasma.

The project's approach is to provide from the beginning diagnostics capable to work under full heating power in steady state plasma discharges and which are designed to sustain ECRH stray radiation. This holds even more so for components which are deeply integrated into the machine, like components located between the first wall and the plasma vessel wall.

The plasma heating systems, directly and indirectly, heat the in-vessel components, which in turn need to be designed to withstand these heat loads under steady state conditions $[13,14]$. In addition, components have to withstand electromagnetic forces which may occur during a fast plasma decay (release of bootstrap current) or during a fast discharge of the W7-X magnet system. Diagnostics therefore need to be designed to withstand the thermo-mechanical and/or electromagnetic forces. In-vessel components not only have to withstand these specified heat loads but furthermore also operation scenarios used for conditioning of the machine, like baking, glow discharge cleaning, and boronisation. In these cases, the diagnostics are typically protected by shutter systems.

Frequently one has to find the right balance in the design between contradictory requirements. For example, a higher thermal conductivity usually goes along with a higher electric conductivity which could be in conflict with an increased electromagnetic force during plasma decay. Any shielding against ECRH stray radiation may cause trapped volumes, which have to be avoided with respect to the vacuum compatibility [15]. A design has to fit into a tight available space with limited access, but on the other hand attachment screws have to be large enough to be handled with gloves or have to be protected against losses during installation. Systems which must be placed close to the vessel but are sensitive to the stray field of the W7-X magnet system have to be shielded by a soft iron box. However, the soft iron itself may introduce an unacceptable disturbance of the magnetic field, where a precision of better than $10^{-4}$ in the plasma volume is required.

The installation phases of W7-X pose a harsh environment, consequently any design has to be robust in this sense. Frequently a diagnostic is quite sensitive or based on filigree components. If so, an adequate protection should be provided during the further assembly work in the vicinity. Step protection, stress relief, protection against accidental contact, and, in particular, a protection of sensitive vacuum feedthroughs must be considered.

Already in the early design phase, the assembly technology must be considered, taking into account assembly and/or metrology tolerances, the assembly sequence, clearance of the transportation route, crane procedure, installation under limited space, and, in constraint posture, scaffolds or platforms and last but not least the safety at work.

In general, each diagnostic project has to undergo a well defined design review process. Before the manufacturing of a component can be released, the aims of a project have to be clearly specified, concepts for realization have to be worked out, the selected concept has to be qualified, safety aspects have to be identified and respective safety measures have to be defined, the design has to be concretized, presented to the overall W7-X project and successfully defended. After this procedure a design can be finalized and the procurement can be initiated.

If a diagnostic is mandatory for operation of $\mathrm{W} 7-\mathrm{X}$, higher demands regarding reliability and/or redundancy are applied. In order not to endanger the operation of W7-X, high reliability is requested for some components. For instance, if a failure of a shutter used to protect in-vessel diagnostic results in the shutter being blocked in the wrong position, plasma operation or plasma vessel conditioning might not be possible until the failure has been fixed.

\section{Quality and management issues}

In a large and complex project like W7-X, quality management and quality assurance are essential [16]. Distinct process flows, mandatory and well-defined proofs and inspections, as well as mandatory certificates for materials or components help the responsible officers of a system to deliver their components in compliance with the W7-X requirements. For example, the cleaning and assembly procedures to fulfill the vacuum requirements are stipulated. Components have to pass an integral leak test successfully, and an acceptable outgassing under operating temperature has to be verified before installation onto W7-X. The processes to cope with unforeseen situations are well defined. Management systems for non-conformity $[16,17]$, change, configuration [18], interface and earned value [19] have been established within the $\mathrm{W} 7-\mathrm{X}$ project.

\section{Set of diagnostics}

\subsection{Divertor gas injection system}

Beside the main W7-X gas injection system a divertor gas injection system was developed mainly for diagnostic purpose. With the divertor gas injection system pulses $(\geq 10 \mathrm{~ms})$ of various gases can be provided at pressures in the range of 10 mbar to 60 bar. In OP 1.2 the diagnostic is installed in one upper and one lower divertor and consists of 5 piezo valves each, a central gas supply, two gas treatment stations and the corresponding spectroscopic observation systems (section 4.8). 
The first version of this diagnostic was already used in OP1.1. For the following operation phases upgrades were necessary. The use of explosive gases such as hydrogen and methane had to be made possible. Safety measures had to be adapted accordingly (i.e. leak detection, safe waste gas treatment, explosion protection). The design of the valve boxes was modified to improve the leak tightness and maintainability (Fig. 1). The box is actively water cooled to limit the maximal temperature at the piezo valves to $70{ }^{\circ} \mathrm{C}$. Due to relatively small driving forces and to ensure $10 \mathrm{~ms}$ pulses, it was essential to keep the mass of the mounting clamp with piston of the individual valves as low as possible. The deformation of the box at maximal operation pressure and at maximal temperature is in an acceptable range.

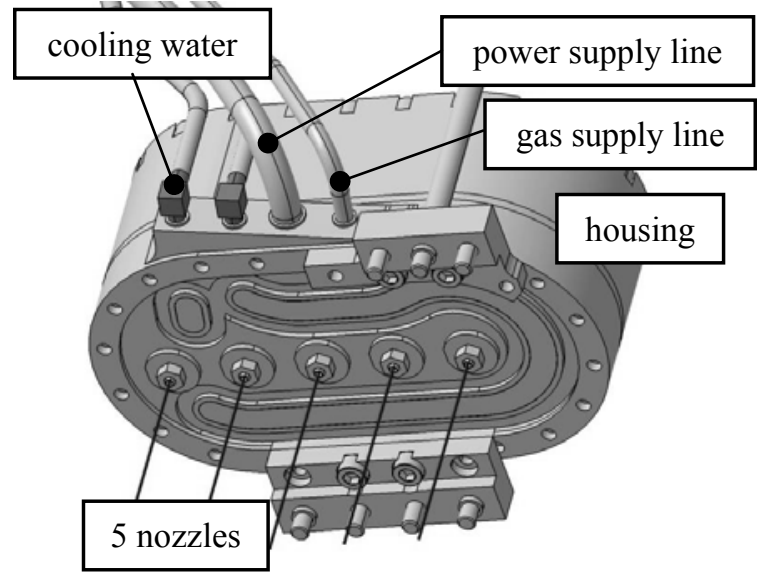

Fig. 1. Valve box of the divertor gas injection system. It is directly attached to the backside of a divertor module and nozzles are inserted though $3 \mathrm{~mm}$ holes to the front side of the divertor.

\subsection{Divertor thermography and $\mathrm{H}$-alpha diagnostics}

A set of cameras for the visible and infrared spectrum was operated in OP1.1 inside immersion tubes in each half module of W7-X. Problems with overheating of the cameras motivated an improvement to the cooling scheme, especially for a new type of infrared cameras. Wide angle optics for each camera now provides a full coverage of the TDU. New narrow spectral filters for $\mathrm{H}$ alpha, H-gamma, CII and CIII lines allow now higher image quality. A new data acquisition infrastructure allows for the online monitoring of the divertor surface temperature and for tests of a real-time image analysis for the protection of the divertor against overheating.

In one half module a first prototype endoscope with a visible and infrared camera is installed for OP1.2a [20]. Such endoscopes will in the future replace all immersion tube systems since they are better suited to the plasma and heating conditions of OP2 and enable steady state operation.

\subsection{Langmuir probes}

In OP1.1, Langmuir probes had been integrated into one of the limiters [5]. For OP1.2 Langmuir probes have been integrated into one upper and one lower divertor unit. They cover most of the width of the horizontal target plate (Fig. 2). The probe bodies are manufactured from the same graphite as the surrounding TDU target \# surface. Since the interpretation of Langmuir probe characteristics is notoriously difficult for incident angles below $3^{\circ}$ [21], the surface of the probes is individually machined with facets (Fig. 2) to have incident angles of $3-6^{\circ}$ for all magnetic configurations, accepting the higher thermal loads relative to the surrounding target surface. Leading edges at the $1 \mathrm{~mm}$ gaps between probe bodies and surrounding targets were avoided by a precise assembly of the probes into the targets.

The section of the signal cables attached to the probes may reach high temperatures. We therefore chose a design with bare $\mathrm{Cu}$ wires in silicate mesh sleevings, inserted into $\mathrm{Cu}$ tubes for electric shielding (Fig. 2). Kapton $^{\circledR}$-insulated twisted-pair cables protected in a copper tube against ECRH stray radiation are used for the more distant in-vessel sections of the signal cables.

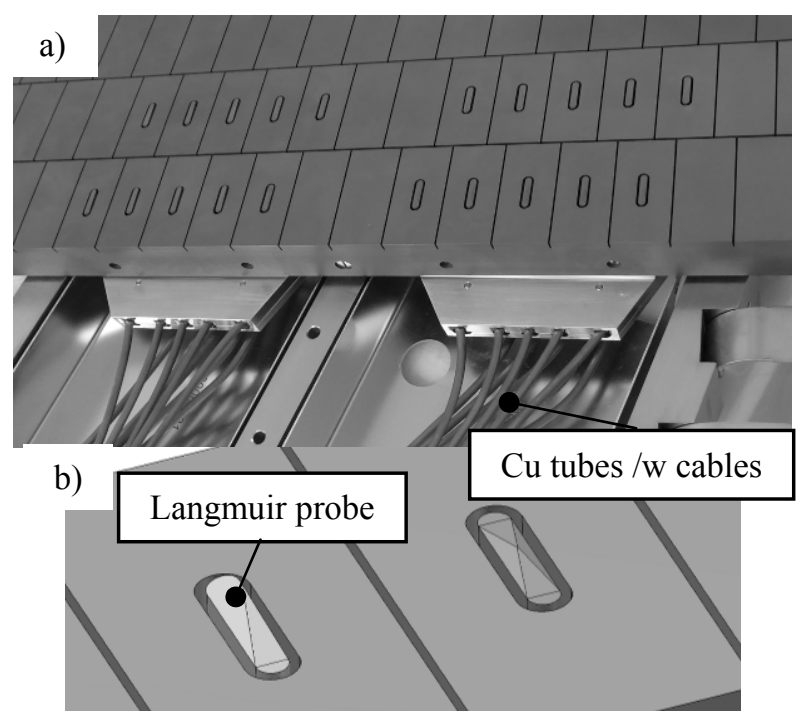

Fig. 2. a) The high-temperature section of the signal cables is visible at the bottom of the photograph. $a+b$ ) Langmuir probes with faceted surfaces integrated into target elements. The facets are individually designed.

\subsection{Video diagnostic}

In OP1.1 the visible video diagnostics (ten tangential views symmetrically distributed in the toroidal direction) was one of the key diagnostics not only for plasma monitoring but also for physics investigations. During OP1.1 the technical weaknesses and the necessary enhancement directions were also revealed and the necessary changes implemented for OP1.2. The monitoring system (EDICAM) software was upgraded with real-time first wall monitoring/protection capabilities. The EDICAM cameras are now capable to study fast phenomena as well by monitoring four small regions of interest in parallel with a framerate up to $40 \mathrm{kHz}$. The system remote control and camera cooling was also extended for safer operation. Beside these cameras three ultra-fast framing cameras (up to $1 \mathrm{Mframe} / \mathrm{s}$ ) are also available at three different toroidal positions for turbulence studies and cryogenic pellet observation.

\subsection{Coherence imaging spectroscopy}

The Coherence Imaging Spectroscopy (CIS) system is a 2D interferometer capable of measuring impurity flow 
velocities via the Doppler effect. Two CIS systems have been installed. Both look at the gas injection system in a lower divertor unit from nearly perpendicular observation directions. The combination of two 2D systems allows obtaining 3D information, while toroidally well localized measurements are possible by using the divertor gas injection system. The systems are placed in the torus hall, due to technical constraints on the image guide length. This implies the need of magnetic shielding. Being the first application on a large stellarator, the CIS diagnostic of W7-X has been designed to be as flexible as possible by including remote controlled motorized components. Another important feature of the W7-X CIS system is its calibration system based on a tunable laser [22].

\subsection{Neutral pressure gauges}

For OP1.2 13 additional pressure gauges have been mounted behind the divertor targets and baffles. Together with the five pressure gauges in midplane positions from OP1.1 [23], the neutral compression ratio of the divertor can be studied. One of the midplane pressure gauges had to be repaired after OP1.1. Since we have in every stellarator module at least one pressure gauge, the machine symmetry can be checked.

Three, for optical spectroscopy optimized Penning gauges [24], have been mounted in the divertor pumping gaps and the midplane of one module. The Penning discharge, with an anode potential of $2 \mathrm{kV}$, is observed with a set of high temperature, ultra-high vacuum optics and analyzed by the filterscope system (section 4.8). This set-up will provide non-invasive measurements of the total as well as partial pressures and is currently setup for He exhaust studies in the island divertor.

\subsection{Alkali metal beam diagnostic}

An alkali metal beam $(60 \mathrm{kV}, 2 \mathrm{~mA}, \varnothing 2-3 \mathrm{~cm})$ [25], which can be either operated with a $\mathrm{Li}$ or a $\mathrm{Na}$ source has been installed in the W7-X midplane, toroidally at one of the up-down symmetric cross sections. For OP1.2 already a fast observation system has been installed perpendicular to the beam. The alkali beam emission along a $20 \mathrm{~cm}$ plasma edge region is imaged onto a $1 \mathrm{D}$ 40 channel linear APD (Avalanche Photo Diode) detector system $(6 \mathrm{MHz}, 14 \mathrm{bit})$. This system will allow for the determination of radial edge density profiles with a radial resolution of $\sim 5-10 \mathrm{~mm}$ at a time resolution of up to $10 \mu \mathrm{s}$. Plasma turbulence measurements in the edge and scrape of layer region are also addressed. With fast $(250 \mathrm{kHz})$ hopping of the beam a quasi-2D resolution is provided which can yield the poloidal flow velocity of turbulence.

\subsection{Visible spectroscopy, $Z_{\text {eff, }}$ and single line of sight observation}

The visible spectroscopy is concentrated in one upper and one lower divertor module with identical observation systems. At both these locations are also the divertor gas injection systems located (section 4.1), which can as well be operated as thermal He- or Ne-beam diagnostic for temperature and density profile measurements. In each module one system looks radially and one vertically at the same divertor cross section with several rows of fibres. For spectral analysis various spectrometers from low to very high resolution will be used.

Till OP2 all 4 observation systems (developed by Forschungszentrum Jülich) will be stepwise replaced by scanning endoscope systems compatible with quasicontinuous operation, which will each be equipped with 5 visible light cameras with interference filters, 2 MIR cameras and various rows of optical fibres for detailed spectral analysis. The cameras will cover the entire divertor region.

The filterscope system with 4 channels per line of sight (1.o.s.) has been upgraded from 6 to 14 l.o.s., further 4 fibres are connected to 4 Penning gauges (section 4.6). 12 l.o.s. are looking through the W7-X midplane at various torodial locations from the inboard and outboard side and 2 radially across the divertor. Each of them can alternatively also be connected to any of the spectrometers.

The 26 optical fibres of the $Z_{\text {eff }}$ Bremsstrahlung profile measurement system will provide a half diameter $Z_{\text {eff }}$ profile. The fibres are connected to a high throughput $(\mathrm{f} / \mathrm{D}=1.4)$ transmission grating spectrometer equipped with a CCD (Charge-Coupled Device) camera, providing spectra from 800 to $1060 \mathrm{~nm}$.

\subsection{Flux surface measurement}

For the flux surface measurements at W7-X a special diagnostic has been set-up, based on the electron beam technique and an optical detection system. The positioning and movement of the electron beam and the detector are realized by remotely controlled manipulators [26]. Already in OP1.1 it could be shown that the magnetic surfaces needed for good confinement is indeed realized, and that the measured deviations are smaller than one part in 100,000 . It was thus demonstrated that the complicated and delicate magnetic topology can be created and verified with the required accuracy [27]. After OP1.1, a third manipulator has been added, increasing the accessible area and giving more redundancy to the flux surface measurement system. The electron guns were modified to improve the electrical strength of the system.

\subsection{TDU scraper element diagnostics}

In OP1.2b scraper elements will be installed in front of one upper and one lower divertor unit [11]. The aim of these scraper elements is to investigate the ability to shield the edges of the divertor on one hand and the detrimental influence on the pumping efficiency of neutral particles at the pumping gap on the other hand. The scraper elements will be equipped with a set of different diagnostics (neutral gas pressure gauge, Langmuir probes, temperature sensors and plasma-wall interaction marker coatings). Since the installation phase between OP1.2a and OP1.2b has to be as short as possible, the assembly technology of the scraper elements was successfully qualified before OP1.2a. Furthermore, the invessel wiring for the different diagnostics behind the first 
wall required from a connector box to the vacuum feedthrough was already prepared before OP1.2a. For OP1.2b camera systems will be complemented to allow infrared as well as visible observation of the scraper elements during plasma operation.

\subsection{Plasma-wall interaction}

Different probes have been installed for the erosion/deposition investigations in three areas with largely different expected heat load levels [28]. These include 18 exchangeable target elements at the TDU, about 30,000 Ti-Zr-Mo screws at graphite tiles and 44 wafer probes on stainless steel wall panels coated with marker layers. The layer thicknesses, surface morphologies and the impurity contents were pre-characterized and subjected to various qualification tests including mechanical and thermal tests. The positions of these probes were decided based on the strike point locations on the divertor for the defined plasma configurations and availability of space on panels with a direct plasma view. After OP1.2a the probes will be partially removed to determine the erosion/deposition pattern and will be replaced with another set for OP1.2b.

Table 1. The set of edge, divertor and scrape-off layer diagnostics for W7-X during the operation phase with inertially cooled divertor units. Column "Modifi." indicates the kind of performed modification ( $\mathrm{U}=$ upgraded, $\mathrm{O}=$ optimized and $\mathrm{N}=$ new diagnostic) compared to OP1.1.

\begin{tabular}{|c|c|c|}
\hline Diagnostic & Modifi. & Remarks \\
\hline Divertor gas injection system & $\mathrm{O}$ & valve box improved; usage of explosive gases now possible \\
\hline Divertor thermography & $\mathrm{O}, \mathrm{N}$ & $\begin{array}{l}\text { IR cameras exchanged; cooling improved; first prototype } \\
\text { system for steady state operation }\end{array}$ \\
\hline H-alpha diagnostic & $\mathrm{O}$ & spectroscopic filters and cooling improved \\
\hline Langmuir probes & $\mathrm{N}$ & in OP1.1 Langmuir probes located in limiter, now in divertor \\
\hline Video diagnostic & $\mathrm{U}$ & camera set upgraded, number of fast cameras increased \\
\hline Coherence imaging spectroscopy & $\mathrm{N}$ & \\
\hline Neutral pressure gauges & $\mathrm{U}, \mathrm{O}$ & more systems; new design for Penning system \\
\hline Alkali metal beam diagnostic & $\mathrm{N}$ & \\
\hline Visible spectroscopy & $\mathrm{U}, \mathrm{O}, \mathrm{N}$ & $\mathrm{U}$ and $\mathrm{O}$ and first system for steady state operation \\
\hline$Z_{\text {eff }}$ & $\mathrm{U}, \mathrm{O}$ & \\
\hline Single line of sight observation & $\mathrm{U}, \mathrm{O}$ & number of systems increased from 4 to 18 ; filters improved \\
\hline Flux surface measurement & $\mathrm{U}, \mathrm{O}$ & number of systems increased from 2 to 3 ; e-guns optimized \\
\hline TDU scraper element diagnostics & $\mathrm{N}$ & \\
\hline Plasma-wall interaction & $\mathrm{N}$ & \\
\hline
\end{tabular}

\section{Acknowledgments}

This work has been carried out within the framework of the EUROfusion Consortium and has received funding from the Euratom research and training programme 2014-2018 under grant agreement No 633053. The views and opinions expressed herein do not necessarily reflect those of the European Commission.

\section{References}

[1] Bosch, H.-S., et al., Nucl. Fusion 53 (2013) 126001.

[2] Warmer, F., et al., Plasma Phys. Control. Fusion 58 (2016) 074006.

[3] Nührenberg, J., Phys. Lett. A 114 (1986) 129.

[4] Grieger, G., and Milch, I., Phys. Bl. 49 (1993) 1001.

[5] Pedersen, T. S., et al., Nucl. Fusion 55 (2015) 126001.

[6] Klinger, T., et al., Plasma Phys. Control. Fusion 59 (2017) 014018.

[7] Pedersen, T. S., et al., Phys. Plasmas 24 (2017) 055503.

[8] Krychowiak, M., et al., Rev. Sci. Instrum. 87 (2016) $11 \mathrm{D} 304$.

[9] Peacock, A., et al., Fusion Eng. Des. 84 (2009) 1475.

[10] Klinger, T., et al., Fusion Eng. Des. 88 (2013) 461.

[11] Fellinger, J., et al., Fusion Eng. Des. 124 (2017) 226.

[12] König, R., et al., J. Instrum. 10 (2015) P10002.
[13] Boscary, J., et al., Fusion Eng. Des. 86 (2011) 572.

[14] Peacock, A., et al., IEEE Trans. Plasma Sci. 42 (2014) 524.

[15] Hathiramani, D., et al., Fusion Eng. Des. 88 (2013) 1232.

[16] Vilbrandt, R., Bosch, H.-S., and Feist, J.-H., IEEE Trans. Plasma Sci. 42 (2014) 3644.

[17] Vilbrandt, R., Bosch, H.-S., and Eeten, P. v., Fusion Eng. Des. 88 (2013) 2151.

[18] Brakel, R., et al., Fusion Eng. Des. 84 (2009) 505.

[19] Lorenz, A., Bosch, H.-S., and Küttler, K., IEEE Trans. Plasma Sci. 40 (2012) 3560.

[20] Chauvin, D., et al., $27^{\text {th }}$ IEEE Symposium on Fusion Engineering (SOFE), submitted for publication in IEEE Trans. Plasma Sci. (2017).

[21] Weinlich, M., and Carlson, A., Phys. Plasmas 4 (1997) 2151.

[22] Perseo, V., et al., $44^{\text {th }}$ EPS Conference on Plasma Physics (2017), http://ocs.ciemat.es/EPS2017PAP/pdf/P5.103.pdf.

[23] Wenzel, U., et al., JINST 12 (2017) C09008.

[24] Flesch, K., et al., Rev. Sci. Instrum. 87 (2016) 11E529.

[25] Anda, G., et al., $41^{\text {st }}$ EPS Conference on Plasma Physics (2014), http://ocs.ciemat.es/EPS2014PAP/pdf/P4.006.pdf.

[26] Otte, M., et al., Plasma Phys. Control. Fusion 58 (2016) 064003.

[27] Pedersen, T. S., et al., Nat. Commun. 7 (2016) 13493.

[28] Dhard, C. P., et al., Phys. Scr. T170 (2017) 014010. 